\title{
Indigenous Languages: A Challenge or an Opportunity in the Achievement of Universal Healthcare in Kenya
}

\author{
Emily Ayieta Ondondo \\ Department of Linguistics, Languages and Literature, School of Humanities and Social Sciences, Jaramogi Oginga Odinga University of \\ Science and Technology (JOOUST), Bondo, Kenya
}

Email address:

eondondo@gmail.com

To cite this article:

Emily Ayieta Ondondo. Indigenous Languages: A Challenge or an Opportunity in the Achievement of Universal Healthcare in Kenya. Communication and Linguistics Studies. Vol. 6, No. 2, 2020, pp. 34-39. doi: 10.11648/j.cls.20200602.14

Received: May 13, 2020; Accepted: June 15, 2020; Published: June 28, 2020

\begin{abstract}
One of the development agenda for Kenya is the achievement of universal healthcare. An assessment of the level at which this has been implemented reveals that Kenya is facing challenges in implementing this endeavor. Health professionals communicate with patients to build trust for more effective practice. Failure to communicate strains the relationship, leading to ineffective practice. One of the main obstacles that may lead to failure in communication is differences in language and culture between the doctor and the patient. Majority of the people that universal healthcare targets in Kenya speak indigenous languages as opposed to English and Kiswahili, languages that the healthcare providers speak. Effective communication occurs through a language that the communicants understand best. Health care providers, in Kenya, understand English and Kiswahili best, while their patients understand Kenyan indigenous languages best. So, what language should be used in universal healthcare endeavors? Should it be English, Kiswahili or Indigenous languages? This shows that one of the main challenges facing the implementation of effective healthcare system and intervention in Kenya could be the language of discussions. Using a descriptive design, this paper takes a critical look at the role of Kenya's indigenous languages in achieving universal healthcare goals. It argues that the achievement of effective and sustainable universal healthcare in Kenya can only become possible through the use of indigenous languages as languages of discussions. And that the use of indigenous languages in universal healthcare endeavors in Kenya should not be seen as a challenge but as an opportunity.
\end{abstract}

Keywords: Effective Communication, Indigenous Languages, Universal Healthcare, Challenge, Opportunity

\section{Introduction}

One of the key factors to quality healthcare is communication. Patients communicate their symptoms and situations to healthcare providers and the healthcare providers in turn relay their findings and recommendations to patients. Effective patient communication can improve adherence to treatment, increase patient satisfaction and lead to better outcomes, which adds up to better overall health. Communication occurs through language [1-3]. Language as a mode of human communication can be either spoken or written and consists of the use of words in a structured and conventional manner. Language and literacy are related. For instance, oral literacy entails the acquisition and mastery of listening and speaking skills, while print literacy involves the acquisition and mastery of writing and reading skills. For many Kenyans oral and print literacy in English and Kiswahili are attained through formal education. Though print literacy in indigenous languages in Kenya needs formal education, oral literacy, to the native speakers of the indigenous languages in question, does not need formal education. To be literate in a language implies to know how to speak it, listen to/and in it, read it and write it. From a linguistic point of view, however, spoken language proceeds written language and any native speaker of a language whether literate or illiterate has the ability to speak and listen to/and in his/her native language [4-10].

Effective communication is achieved through the use of a language that the communicants know and understand best [1-3]. That is a language that they both have, at least, oral literacy in. In this way, quality health care can only be achieved when healthcare providers and their clients have 
shared oral literacy in the language of healthcare endeavors. This common ground becomes handy in facilitating and maintaining health literacy. Health literacy can be defined as the degree to which individuals have the capacity to obtain, process and understand basic health information needed to make appropriate health decisions $[11,12]$. This implies that limited language skills are associated with worse health outcomes.

This study assesses the extent to which indigenous languages, which majority of the Kenyans have oral literacy in, are used in healthcare provision in Kenya. It argues that the achievement of effective and sustainable universal healthcare in Kenya can only become possible through the use of indigenous languages as languages of discussions. The use of English and Kiswahili is a barrier to effective communication in healthcare endeavors and makes the whole process alien. Therefore, the use of indigenous languages in universal healthcare endeavors in Kenya should not be seen as a challenge but as an opportunity.

\section{Indigenous Languages, Indigenous Knowledge and Identity}

Each and every ethnic community in Kenya is tied to a particular indigenous language and culture that its members identify with. Indigenous languages are also the store and carriers of indigenous knowledge [13]. Linguistic, cultural and indigenous knowledge aspects of a given community affect how they perceive, internalize, react to and embrace issues, health issues included. The use of alien languages, therefore, comes with alien linguistic, cultural and knowledge aspects that would affect how communities in Kenya embrace healthcare issues. The use of indigenous languages, with their rich culture and indigenous knowledge in healthcare endeavors would, therefore, go a long way in facilitating the achievement of universal and quality healthcare. These languages are familiar to the majority of the people to whom universal and quality healthcare is to be provided. Majority of these people also identify with and embrace these languages because these are the languages that they have oral literacy in and also because they are the languages that they use in their daily today activities. However, the linguistic diversity in Kenya would be seen as a big challenge to the use of indigenous languages in healthcare activities. There are over forty-two indigenous languages spoken in Kenya, which are specific to communities. To use these languages in healthcare provision, healthcare personnel would need to have both oral and print literacy in these languages. This would call for the introduction of such languages as examinable subjects in healthcare personnel training. The achievement of such a target would be seen as farfetched in the Kenyan context given the logistics involved. Therefore, the most apt alternative is the use of English and Kiswahilli, languages whose literacy development, both oral and print, is ingrained in the formal training program of health personnel in Kenya.

\section{Universal Healthcare in Kenya}

The health field is a contested space where alternative conceptions of illness and treatment compete with the dominant tradition of scientific medicine [14]. There is an unwritten contract between the health personnel and the patient. The health personnel's technical expertise entitles $\mathrm{him} /$ her to the institutionally ratified role of a 'healer', while the patient takes on the role of the 'sick,' which he/she accepts. The 'sick' role is characterized by exemption from normal social responsibility and the obligation to seek technically competent help in order to achieve the goal of recovery [15]. The features of the 'healer' role are objectivity, effective neutrality and technical competence, while those of the 'sick' role are helpless dependence, emotional involvement and technical incompetence [15]. In this way the health personnel have a superior role over the patient. The patient in such a framework is dominated by the health personnel and has little or no say in what ensues in any healthcare endeavors he/she is involved in.

However, there is a departure from the tradition as described in the preceding discussion. There has been a shift from a patient being a dependent outsider towards an increase in lay involvement in medical decision-making and an acknowledgement of the value of the individual's subjective experience of illness [16]. In this case, the 'sick' role has been replaced by the 'health' role in which the individual assumes responsibility for their health maintenance through appropriate lifestyle choices, thereby reducing the centrality of the health personnel. This then implies that the health personnel and the patients have specific equal roles that they play in healthcare activities and no role is superior to the other and none of the roles should be overlooked or undermined. In this way, the health personnel and their patients enter into healthcare activities as equal partners with equal rights. In Kenya, the trend towards increased lay involvement was reflected by the introduction in 2009 of the patient's charter as stipulated in the Ministry of health strategic plan, 2008-2012, a document which was designed to redress the clinical balance in favor of patients and involve them in medical decision making at all levels thereby envisioning successful implementation of the universal healthcare coverage.

Universal healthcare coverage, as postulated and intended by Kenya's development agenda involves availing certain priority health services to all Kenyan citizens without their need to pay at the point of use. It also entails ensuring that the services provided are of good quality and meet the users' expectations. This means that universal healthcare coverage involves all the healthcare personnel at healthcare facilities. Given that all healthcare personnel at healthcare facilities, in Kenya, primarily use language to transact their business. It is important to assess the language used by healthcare personnel in healthcare endeavors in order to determine whether it facilitates access or impede medical access which eventually affects the universal health care coverage. The driving principle here is that universal healthcare coverage, as one of Kenya's big four development agenda, requires that health 
services be patient oriented and meet the patient's expectations at the point of contact.

\section{Indigenous Languages and Healthcare}

Healthcare endeavors and activities in Kenya are predominantly carried out through English and Kiswahili, and rarely through Kenyan indigenous languages. Indigenous languages are only used in situations where the health personnel and the patients share an indigenous language. This is because, in Kenya, indigenous languages are neither used as official nor national languages, positions taken by English and Kiswahili respectively. They are only used at the family and community levels. Similarly, literacy in indigenous languages in Kenya is still on the minimal compared to literacy in English and Kiswahili. As a result, the many community members using these languages and to whom only such languages are known are not able to contribute significantly in healthcare interactions due to their illiteracy in English and Kiswahili and the overall illiteracy in indigenous languages in Kenya. Given that shared language between patients and healthcare providers enables gathering information to arrive at diagnoses, explaining treatment strategies, and ensuring understanding and joint decision-making, the role of indigenous languages in achieving sustainable universal healthcare coverage cannot be ignored.

In Kenya, the ability to speak one's indigenous language, is a key component of ethnic identity and is, for some, indicative of a true Luo, Kikuyu, Kamba or Kisii for instance. In healthcare, in Kenya, speaking a common language is central to good communication and is crucial in giving and obtaining quality services and appropriate care. The relationship between the ability to speak a common language and the quality of health care given and received has been established by research linking limited language proficiency with less care-giving/care-seeking, diminished quality of and access to health services, poorer health outcomes, and even death [17-22].

Elements of language enable thoughts and feelings to be conveyed. Linguists and cognitive scientists argue that language influences how people think and view the world $[4-10,23,24]$. Language is, thus, a window on how the human mind works $[25,26]$. Mental and social constructs are embedded in language to describe thoughts, interpret meaning, and interact with others [20, 25]. In this sense, indigenous languages and the culture and knowledge they carry determine how healthcare seekers in Kenya view, react to and engage in healthcare interactions given that these are the languages that they know and identify with. If other languages are used besides the indigenous languages that healthcare seekers know well, the whole interaction will be unfamiliar and without the desired outcome due to failure to communicate effectively. As this affects the description of thoughts, interpretation of meaning, and how the interactions between the healthcare givers and the healthcare seekers are conducted and facilitated.

Effective communication is communication that is comprehended by both participants in a communication event [27-29]. It is usually bidirectional between participants, and enables both participants to clarify the intended message. In the absence of comprehension, effective communication does not occur and when effective communication is absent, the provision of healthcare ends or proceeds only with errors, poor quality, and risks to patient safety [30-32]. Schyve argues that when patients with limited English proficiency are treated by physicians and other health professionals who are proficient only in English, three factors converge to create a 'triple threat' to effective communication [31]. First, the language differences themselves are a barrier to effective communication. This, however, is not noticed immediately because the healthcare giver and the healthcare seeker often underestimate the language barrier between them.

Second, the language differences come with cultural differences, which are a barrier to effective communication. One's culture affects one's understanding of a word or a sentence and even one's perception of the world. This is because to learn a language is not the same as understanding a culture, even those who share a common native language may not share a common culture. And not everyone born in the same place and speaking the same language necessarily shares all the features of a common culture. Therefore, there is a risk of either underestimating the effect of cultural differences or of stereotyping individuals by their culture. And these will interfere with the effectiveness of communication [31].

Third, low health literacy is a barrier to effective communication. Low health literacy in patients may be associated with language and cultural barriers [11]. Nonetheless, it is also witnessed in patients who are proficient in a given language and who share a common culture. Where there are differences in language and culture, the situation is clear. The healthcare giver here only explores whether the healthcare seeker understands the oral or written communication of the healthcare seeker. The situation, however, is tricky with the latter group. This is because such a group is at risk of having their low health literacy go unrecognized, for the fact that it is assumed that if they speak the same language and share a culture then there are no language and cultural barriers. But it is ignored that low health literacy may be a barrier. And any competent speaker of any language is likely to suffer from low health literacy. That is, he/she may not be able to read or write and worse still he/she may not be in a position to understand the healthcare giver medical jargon or complex instructions that constitute health literacy [31].

The healthcare personnel in Kenya predominantly use English in their discussions with patients. Sometimes, they also use Kiswahili or they code switch between English and Kiswahili. Rarely do they use indigenous languages in their discussions with patients. The use of English and Kiswahili brings with it language and cultural barriers, given that majority of the people at the grassroots and in need of healthcare speak indigenous languages. In this case effective communication is adversely effected, resulting into poor 
services and limited achievement of universal healthcare coverage. In addition to this, health literacy is a major barrier. The healthcare givers and seekers are not only faced with language and cultural barriers but also health literacy barriers. The healthcare seekers are not able to read and write English. They are also not in a position to understand the medical jargon and complex instructions given to them in English and/or Kiswahili. On the other hand, the healthcare givers are not able to read and write or give instructions in indigenous languages. The healthcare seekers, then result to exploiting their indigenous systems and knowledge to treat themselves instead of seeking advice from healthcare providers. This then jeopardises the achievement of universal healthcare in Kenya.

In a multilingual, multicultural society like Kenya, providing safe, high-quality universal healthcare requires overcoming these 3 barriers to effective communication with patients and their families. Today, effective communication, which takes into account language, cultural differences, and health literacy, is seen as a prerequisite to safe and universal healthcare. This is because communication problems are the most frequent root cause of serious adverse events in healthcare provision. When patients suffer adverse outcomes from medical errors, the outcomes are more serious in limited language proficiency patients than in patients with good language proficiency. Effective communication is, therefore, necessary for the observation and achievement of patient rights, quality care, and patient safety. These together make effective communication in health care obligatory and as a critical component of the healthcare itself.

There is, therefore, need to embrace the use of indigenous languages as a common ground. Indigenous languages are used by the majority of the healthcare seekers. They are also closer to them in terms of knowledge, thought, culture and identity. Because these languages are familiar to healthcare seekers, it will not be difficult for them to understand and comprehend healthcare discussions. It will also make healthcare interactions easy and smooth. This will then facilitate and make easy the embracement, ownership and adoption of any healthcare issues presented to them. This will in turn facilitate the achievement of universal healthcare.

The immediate and more effective mechanism of integrating the use of indigenous languages into the healthcare system is to incorporate a language interpretation service into redesigned work processes. In the beginning, the medical jargon may be a challenge in terms of equal and one to one translation from English and/or Kiswahili to the target indigenous languages. However, inventions, nativisations and coinages are not farfetched. With time these can be developed into a full blown jargons ingrained in the specific indigenous languages. This will in the long run not only alleviate general literacy in these languages but also health literacy, which is also a big problem when English and/or Kiswahili is used with the healthcare seekers in the rural areas whose level of competence and understanding of English and/or Kiswahili are not only low but the languages are also foreign to them. The interpreters should be people from the same community and having resided in the community for the same time as the majority of the community members to guard against the cultural difference barrier.

Though it seems challenging to incorporate indigenous languages into healthcare discussions and interventions, if all the efforts discussed above are put in place, this will provide an opportunity to help develop oral and written literacy in these languages both for the native and non-native speakers of these languages. This is an opportunity for Kenya as it will go further in enhancing the use of these languages in other spheres of national development, given that these languages as discussed above are the engines driving community life, which embraces both social economic and development issues. In this way, this will enable these communities and Kenya as a whole to attain development in all spheres including the health sector.

\section{Conclusion}

The management of cultural and linguistic barriers in the field of medicine can be challenging due to the patients' language, culture, traditions, family background and health beliefs. A lack of effective communication hinders the medical process and the provision of healthcare. Therefore, healthcare personnel must acquire specific knowledge and skills known as cultural competence. The doctor should attempt to ensure quality in the health care communication services provided for diverse patients. In recent times, emphasis is being placed on the interaction between doctors and patients as poor communication leads to complaints and misunderstandings. Good communication is essential to effective practice as it assists healthcare professionals to identify the core elements of this process. A good physician must know how to ask about and handle the patient's situation. He/she needs to explain the results in a way that patients can understand and remember. $\mathrm{He} / \mathrm{she}$ also needs to encourage the patients to express their fears and concerns. But in order to achieve all these, the doctor must master the target language and be able to simplify the medical jargon to the patients. He/she should explain medical terminology to the patient and answer the patient's colloquial questions about prognosis of the disease [33-39]. This necessitates the use of a common language between the doctor and the patient. The benefits of good communication are that it builds trust during the conversation, helps the patient open up and enhances his satisfaction, helps the patient take good decisions and have realistic expectations, improves the medical process and cuts down the risk of mishaps. Poor communication, on the other hand, reduces confidence in the medical act, hinders the patient from disclosing essential information, causes patient distress, misunderstandings and misinterpretation of medical advice. This can also predict negligence claims and determine the patient to give up on treatment or medical care. Therefore, physicians must do their best to decrease obstacles to good communication. They must take into account whether the patient needs an 
interpreter. The interpreter may be a professional interpreter or family members or friends can also be used to carry out the process of interpreting. In the Kenyan context this calls for the embracement of Kenyan indigenous languages in the healthcare system by the use of interpreters to begin with.

\section{References}

[1] Altbach, P. G. (1999). The Dilemma of Publishing in African Languages: A Comparative Perspective. In P. G. Altbach \& D. Teferra (Eds.). Publishing in African Languages: Challenges and Prospects. Bellagio Studies in Publishing 10, 1-10. Chestnut Hill, MA: Bellagio Publishing Network.

[2] Kembo-Sure. (1991). Language Functions and Language Attitudes in Kenya. English World Wide Language and Communication. Cambridge: MIT Press.

[3] Ogechi, N. (2001). Publishing in Kiswahili and Indigenous Languages for Enhanced Adult Literacy in Kenya. Afrikanistische Arbeitspapiere 68 (2001)-Swahili Forum 3, 185-199.

[4] Chomsky, N. (1986). Knowledge of Language. Cambridge: MIT Press.

[5] Crystal, D. (2010). The Cambridge Encyclopedia of Language. Cambridge: Cambridge.

[6] Fasold, R. (1992). The Sociolinguistics of Society. Oxford: Blackwell.

[7] Fromkin, V. R., \& Hyams, N. (2010). An Introduction to Language. Boston: Thomson-Henle.

[8] Lyons, J. (1992). Language and Linguistics: An Introduction. Cambridge: Cambridge University. Wardhaugh, R. (1992). An Introduction to Sociolinguistics. Oxford: Blackwell.

[9] Wardhaugh, R. (1992). An Introduction to Sociolinguistics. Oxford: Blackwell.

[10] Yule, G. (2000). The Study of Language. Cambridge: Cambridge University Press.

[11] Nielsen-Bohlman, L, Panzer, A. M., \& Kindig, D. A. (2004). Health Literacy: A Prescription to End Confusion. National Academies Press.

[12] U.S. Department of Health and Human Services, Office of Disease Prevention and Health Promotion. (2010). National Action Plan to Improve Health Literacy. Washington, (DC).

[13] Wekundah, J. M. (2012). Why Protect Traditional Knowledge, Nairobi Kenya: The African.

[14] Hyden, L-C., \& Mishler, E. (1999). Language and Medicine. Annual Review of Applied Linguistics 19, 174-192.

[15] Parsons, T. (1952). The Social System. London: Routledge and Kegan Paul.

[16] Lawton, J. (2003). Lay experiences of health and illness. Sociology of Health and Illness 25, 23-40.

[17] Cohen, A. L., Rivara, F, Marcuse, E. K., McPhillips, H., \& Davis, R. (2005): Are Language Barriers Associated with Serious Medical Events in Hospitalized Pediatric Patients? Pediatrics 116, 575-579.
[18] Flores, G., Abreau, M., Olivar, M., \& Kastner, B. (1998). Access Barriers to Health Care for Latino Children. Arch Pediatr Adolesce Med 152, 119-125.

[19] Manson, A. (1988). Language Concordance as a Determinant of Patient Compliance and Emergency Room Visits in Patients with Asthma. Med Care 26, 1119-1128.

[20] Partida, Y. (2012). Language and Health Care. Diabetes Spectrum 25 (1), 245-260.

[21] Shapiro, J., \& Saltzer, E. (1981). Cross-Cultural Aspects of Physician-Patient Communication Patterns. Urban Health 10, 10-15.

[22] Woloshin, S., Schwartz, L. M., Katz, S. J., \& Welch, H. G. (1997). Is language a Barrier to the Use of Preventive Services? J Gen Intern Med 12, 472-477.

[23] Aitchison, J. (1992). Linguistics. Oxford: Oxford University Press.

[24] Aronoff, M. \& Reese-Miller, J. (2006). The Handbook of Linguistics. Malden MA: Blackwell.

[25] Clark, A. (2006). Language, Embodiment, and the Cognitive Niche. Trends Cogn Sci 10, 370-374.

[26] Partida, Y. (2011) "The Challenge of Health Insurance Language." Presentation Delivered at an Institute of Medicine Roundtable on Health Literacy in Washington, D. C., 19 July 2011.

[27] Akmajian, A., Demers, R., Farmer, A., \& Harnish, R. (2010). Linguistics: An Introduction to Language and Communication. Cambridge, Mass: MIT Press.

[28] Lunenburg, F. C. (2010). Communication: The Process, Barriers, And Improving Effectiveness. Schooling, 1 (1), $1-11$.

[29] Stanton, N. (2009). Mastering Communication. Palgrave: Macmillan. Technology Policy Studies Network. University Press.

[30] Divi, C., Koss, R. G., Schmaltz, S. P., \& Loeb, J. M. (2007). Language Proficiency and Adverse Events in U.S. Hospitals: A Pilot Study. International Journal of Quality Health Care 19, 60-67.

[31] Schyve, P. M. (2007). Language Differences as a Barrier to Quality and Safety in Health Care: The Joint Commission Perspective. J Gen Intern Med 22 (Suppl 2), 360-1.

[32] Wilson-Stronks, A., \& Galvez, E. (2007). Hospitals, Language, and Culture: A Snapshot of the Nation. Oakbrook Terrace, IL: The Joint Commission.

[33] McCullagh, M., Wright, R. (2010). Good Practice. Communication Skills in English for the Medical Practitioner. Cambridge: Cambridge University Press.

[34] Nápoles, A., Santoyo-Olsson, J., Karliner, L., Gregorich, S. E., Pérez-Stable, E. J. (2015). Inaccurate language interpretation and its clinical significance in the medical encounters of Spanish-speaking Latinos. Med Care, 53: 940-947.

[35] Lion, K. C., Ebel, B. E., Rafton, S., Zhou, C., Hencz, P., Mangione-Smith, R. (2015). Evaluation of a quality improvement intervention to increase use of telephonic interpretation. Pediactrics, 135: 709-716. 
[36] Squires, A. \& Jacobs, E. A. (2016) Language and communication issues impact healthcare providers around the world. International Journal of Nursing Studies, 54: 5-6

[37] Facts about patient-centered communications. Oakbrook Terrace, IL: The Joint Commission; October 2017.
[38] Green, A. R. (2017). Language based inequalities in health care: who is the "poor Historian"? AMA J. Ethics, 19 (3): 262-271.

[39] Karliner, L. S. (2018). When Patients and Providers Speak Different Languages. PSNET. 